\title{
The significance of hypoxia as a molecular and cellular event in patients with toxic and non-toxic goitre: A statistical inference based on cross-sectional analytic of Iraqi patients
}

\author{
Mohammed Assi ${ }^{1,2}$, Samia Elewi ${ }^{1}$, Ahmed Al-Imam ${ }^{3,4,5,6}$, Basem Ahmed $^{7}$ \\ ${ }^{1}$ Department of Anatomy, Histology, and Embryology, College of Medicine, Al-Mustansiriya University, Iraq, ${ }^{2}$ The Royal \\ College of Pediatrics and Child Health, United Kingdom, ${ }^{3}$ Department of Anatomy and Cellular Biology, College of \\ Medicine, University of Baghdad, Iraq, ${ }^{4}$ Department of Postgraduate Medicine, School of Life and Medical Sciences, \\ University of Hertfordshire, United Kingdom, ${ }^{5}$ The Canadian Association for Neuroscience, ${ }^{6}$ The Japanese Association \\ of Anatomists, ${ }^{7}$ Department of Pathology and Forensic Medicine, College of Medicine, Al-Mustansiriya University, Iraq
}

A B S T R A C T

Background: Disorders of the thyroid gland, including toxic and non-toxic goitre, are more common in adult female patients. Hypoxia-inducible factors, including HIF-1 and HIF-2, represent transcriptional activators that function as regulators of oxygen homeostasis. Rapid progress is being made in clarifying the homeostatic functions of HIFs in several physiological systems. However, there are much to be learned in connection with the thyroid gland and its pathologies. Aims and Objectives: To assess the biochemical and clinical significance of hypoxia-inducible factors in patients with multinodular goitre. Materials and Methods: The study is observational and cross-sectional analytic conducted among a population of individuals with diffuse and nodular thyroid goitre including clinically toxic and non-toxic patients. It will attempt to answer the research question concerning the significance of HIFs, via applying techniques of immunohistochemistry to histological samples of resected thyroid tissue, and in pertinence with the demographic and clinical parameters of patients.Results: The total number of patients was forty-three, and most was in their fifth decades of life. The percentile contribution of males and females was $11.63 \%$ and $88.37 \%$ respectively. Individuals with toxic goitres accounted for $13.95 \%$, and those had significantly higher levels of both HIF-1 and HIF-2 than non-toxic patients $(p=0.019, p=0.072)$. Clinically-toxic patients also had notably more elevated levels of HIF-1 as they grew older when compared to non-toxic patients. Conclusion: There is an evident inadequacy of published literature on hypoxia-inducible factors in patients with goitre. There are no studies whatsoever in correspondence to the research questions explored in this study. Future attempts should explore experimental designs while using human as well as animal models and from an interdisciplinary perspective.

Key words: Epidemiology; Public health; Evidence-based medicine; Statistical inference; thyroid gland; Goiter; Hypoxia-inducible factor; Immunohistochemistry; Cross-sectional studies; Case report; Middle east; Iraq

\section{INTRODUCTION}

Thyroid goitre is one of the most common endocrine disorders in Iraqi population, particularly among adult women. ${ }^{1}$ A multinodular goitre (MNG) is a non-tumorous condition although a thyroid cancer can be identified
http://nepjol.info/index.php/AJMS DOI: 10.3126/ajms.v9i5.20597 E-ISSN: 2091-0576 P-ISSN: $2467-9100$ 
The hypoxia-inducible factors (HIFs) are well-known heterodimeric transcription factors that are made of an oxygen sensitive alpha subunit (HIF- $\alpha$ ) and a constitutive beta subunit (HIF- $\beta$ ), and these are known to be strictly associated with the hypoxic microenvironment. ${ }^{4,5}$ Under hypoxic conditions, HIF- $\alpha$ subunits get translocated to the nucleus where they heterodimerize with ARNT (aryl hydrocarbon receptor nuclear translocator) and bind to HREs (hypoxia response elements) located within genomic regulatory components of HIF target genes. After stabilisation, the HIF- $\alpha$ /ARNT complex activates gene expression and transcription by recruiting the transcriptional activators p300 and CBP, and ultimately these changes will increase oxygen delivery and enhance metabolic adaptation to hypoxia. ${ }^{6}$

To date, there are three types of HIF- $\alpha$ subunits, namely HIF- $1 \alpha$, HIF- $2 \alpha$, and HIF- $3 \alpha$, and at present, the HIF- $1 \alpha$ and HIF- $2 \alpha$ gained more attention. ${ }^{7}$ Studies concerning the role of HIFs in thyroid tissue are lacking. There are even fewer studies which looked at the co-expression both isoforms within the same histological specimen. Moreover, there is no one study to date which explores the bio-expression of both isoforms in multinodular goitres. Therefore, in this study, we investigated whether there is any difference in the expression of HIF- $1 \alpha$ and HIF- $2 \alpha$ between clinically toxic and non-toxic patients with multinodular goitres.

\section{MATERIALS AND METHODS}

The study is a cross-sectional analytic with statistical inference. All histological samples and laboratory manipulations, including immunohistochemical processing and staining, were carried out with the full consent of the patients and was approved by the local ethical committee of the College of Medicine at Al-Mustansiriya University in Iraq. The total sample size is forty-three $(n=43)$ that is made of cases of nodular goitre including those with and without manifestations of a clinically-toxic thyroid. Patients were surveyed at AlYarmouk Teaching Hospital in Baghdad during the period from June 2017 to January 2018. Demographic parameters including age and sex were tabulated as well as the status of clinical toxicity for each patient.

All thyroid biopsies were instantly fixed in 10\% neutral buffered formalin for 18-20 hours at room temperature $\left(20-25^{\circ} \mathrm{C}\right)$, and as recommended by the manufacturer kit's leaflet. Following fixation, tissue samples were processed according to Luna (1968) as it is the best method for treating small biopsies of endocrine organs. ${ }^{89}$ Then, samples were labelled and embedded in paraffin as blocks. Paraffin blocks were sectioned into 4-6 $\mu \mathrm{m}$ thick slices using a disposable blade for each block. From each tissue block, two histological sections were collected, one for HIF-1 and the other for HIF2 immunohistochemical staining. The histological sections were baked at $68^{\circ} \mathrm{C}$ for two hours and soaked in xylene.

Antigen retrieval was performed by boiling the slides with citrate buffer $(\mathrm{PH}=6)$ at $95^{\circ} \mathrm{C}$, then blocked by UltraCruz ${ }^{\circledR}$ Blocking Reagent (sc-516214). Later, all slides were submerged in Peroxidase quenching solution for 15 minutes and washed twice with phosphate-buffered saline (PBS) for $7 \mathrm{~min}$. The samples were incubated with an antibody against HIF-1 $\alpha$ (1:100; mouse monoclonal, clone; sc-13515; Santa Cruz Biotechnology; USA) and HIF-2 $\alpha$ (1:100; mouse monoclonal, clone; sc-13596; Santa Cruz Biotechnology; USA) in an automatic immunostainer and kept at $4{ }^{\circ} \mathrm{C}$ overnight. Later, slides were incubated for 90 minutes with m-IgGk BP-HRP (1:50; sc-516102; Santa Cruz Biotechnology; USA), and then treated with Diaminobenzidine (DAB) (sc-24982; Santa Cruz Biotechnology; USA) for $2 \mathrm{~min}$. Finally, the sections were counterstained with Mayer's haematoxylin, dehydrated and mounted in DPX mounting medium.

Histological slides were examined via a multiheaded microscope by two consultant pathologists who were blinded to the clinical data, patients' demographics, and the outcome of the study. The pathologists performed a semi-quantitative assessment of immunohistochemical (IHC) scoring. The final IHC score on a continuous numeric scale of $0-3$, was calculated based on the multiplication of the intensity of IHC staining and percentage of positive IHC signals within each slide. The intensity score was designated as 0 (no staining), 1 (weak staining), 2 (moderate staining), and 3 (strong staining).

Statistical analyses were conducted via SPSS version 24 and Microsoft Excel 2016. The inferences were based on the implementation of Levene's test for equality of variances, Student's t-test, Chi-Square test and Fisher Exact test, as well as Linear regression models. The level-of-evidence for this study ranks well within the pyramid of the hierarchy of evidence-based studies. It is estimated to be of level-3 on the categorisation scheme of level-of-evidence imposed by the Oxford Center for Evidence-Based Medicine as of May 2016. ${ }^{10-12}$

\section{RESULTS}

The total number of participants in this study is forty-three $(\mathrm{n}=43)$. The percentile contribution of males and females was $11.63 \%$ and $88.37 \%$ respectively. Patients with toxic goitres accounted for $13.95 \%$ of the total sample. One statistical outlier was detected and hence excluded from subsequent inferential analytic. Cases were approaching normality of distribution with a skewness of 0.191 . Based on age, cases were distributed into class intervals including 
those of age 19-27 years ( $\mathrm{n}=4), 27-35(6), 35-43(10)$, 4351 (15), 51-59 (5), 59-67 (2), and 67-75 (1). The average values were $43.26+/$ - 11.36 (age), $1.41+/-0.80$ (IHC Score - HIF1), $1.34+$ + - 0.62 (IHC Score - HIF2). IHC scores of HIF-1 averaged $1.39+/-0.79$ for females and $1.57+/-0.92$ for males, while IHC scores of HIF-2 averaged $1.32+/-0.62$ for females and $1.50+/-0.66$ for males. Cases with non-toxic manifestation had an average of $1.31+/-0.78$ (IHC Score - HIF1) and 1.26 +/- 0.60 (IHC Score - HIF2), while cases with toxic manifestations averaged $2.06+/-0.56$ (IHC Score - HIF1) and $1.82+/-0.57$ (IHC Score - HIF2).

Student's t-test was successful in extrapolating an inference based on the stratification by sex and toxicity of clinical manifestations. It has been concluded that there is no significant difference based on sex, i.e. males versus females, and in connection with age (42.20 versus 43.39, $p$-value $=0.718)$, as well as the IHC scoring of HIF-1 (1.57 versus $1.39, \mathrm{p}=0.688)$ and HIF-2 (1.50 versus 1.32 , $\mathrm{p}=0.589)$. Hypothesis testing also confirmed the absence of a significant difference based on the presence-absence of clinical toxicity and in connection with age (46.00 versus $42.58, \mathrm{p}=0.570$ ). However, a significant difference existed in between clinically-toxic and non-toxic cases for IHC scoring of HIF-1 (2.06 versus $1.30, \mathrm{p}=0.019)$ and HIF-2 (1.82 versus $1.28, \mathrm{p}=0.072)$.

Based on linear regression models, it has been established that age is positively correlated with IHC scoring of HIF$1\left(d f=1, R^{2}\right.$ score $=0.158, p$-value $\left.=0.008\right)$. However, it was not related with IHC scoring of HIF-2 ( $d f=1, R^{2}<0.001$, $\mathrm{p}=0.984)$. Nevertheless, there was a strong correlation in between IHC scoring of HIF-1 and HIF-2 $(d f=1$, $\left.R^{2}=0.413, \mathrm{p}<0.001\right)$. Chi-Square test and Fisher's Exact test were implemented to test the significance of odds ratio, as a measure of association, for assessing the exposureoutcome relationship and in connection with quartile distribution of age versus IHC scoring of HIF-1 (odds ratio $=3.21, p$-value $=0.067$ ), age versus IHC scoring of HIF-2 (odds $=0.83, \mathrm{p}=0.768$ ), sex versus IHC scoring of HIF-1 (odds=1.58, $\mathrm{p}=0.457$ ), sex versus IHC scoring of HIF-2 (odds $=0.12, \mathrm{p}=0.040$ ), as well as the presence or absence of toxicity versus IHC scoring of HIF-1 (odds $=0.16$, $\mathrm{p}=0.078)$ and HIF-2 (odds $=0.25, \mathrm{p}=0.120$ ).

To recapitulate, most of the patients were females on their $5^{\text {th }}$ decade of life and clinically non-toxic. Males and females had comparable age and levels of HIF-1 and HIF-2. Based on the measures of central tendency, clinically-toxic and non-toxic patients were somewhat identical in age. However, clinically-toxic patients had significantly higher levels of hypoxia-inducible factors. Besides, clinically-toxic patients had notably more elevated levels of HIF-1 compared to patients with no toxic clinical manifestations. This sort of association holds to be true as patients get more advanced in age. Further, the levels of HIF-1 and 2 appear to be rising somewhat simultaneously in parallel to each other.

\section{DISCUSSION}

\section{Critical appraisal of literature}

Three databases of literature were explored including PubMed-NCBI, the Cochrane Library, and Elsevier (Table1). Keywords based on Medical Subject Headings (MeSH) were used in solitary and in various combinations using Boolean operators (AND, OR, NOT) to retrieve the most relevant hits (manuscripts) concerning the topic of our research. The summative number of hits was 323295 distributed as 322302 (PubMed-NCBI), 71 (the Cochrane Library), and 922 (Elsevier). Two combinations of Keywords generated the most valid hits including "Thyroid Gland AND ("Hypoxia-Inducible Factor" OR HIF)" and "Goiter AND ("Hypoxia-Inducible Factor" OR HIF)".

It is evident that there is a lack of literature when it comes to goitre and hypoxia-inducible factors. Besides, the published body of literature has a complete absence of research attempts originating from the Middle East, specifically from Iraq, and in connection with our research questions. Following the application of inclusion criteria, the total number of reference materials used for the citations and the purpose of a literature review reached twenty-eight. Following the elimination of duplicate articles, the inclusion criteria were based on the selection of papers written in the English language for studies and experimentations on human as well as using animal models, and successfully passing the critical appraisal using CASP appraisal tool. ${ }^{11,12}$ Priority was given to studies published in the past 5-10 years.

\section{Discussion of results and review of literature}

Hypoxia is a common condition found in a wide range of solid tumours and has been accepted to play an essential role in cancer development and progression. ${ }^{13}$ Tumour adaptation to hypoxia depends mostly on HIF- $1 \alpha$ and HIF- $2 \alpha$. Both isoforms are regulated by oxygen-dependent hydroxylation that results in intracellular degradation of proteins by the proteasome pathway. ${ }^{14}$ This study is based on the central hypothesis that HIFs facilitate both oxygen delivery and adaptation to oxygen deprivation by regulating the expression of genes that are involved in many cellular processes, including glucose uptake and metabolism, angiogenesis, erythropoiesis, cell proliferation, and apoptosis. ${ }^{15}$

HIF- $1 \alpha$ and HIF- $2 \alpha$ have a similar structure and function, but they have a different topography of tissue and celltype distributions. ${ }^{16-18}$ Recent data indicate that regulation 


\begin{tabular}{|c|c|c|c|c|}
\hline \multirow[t]{2}{*}{ Keywords (MeSH) } & \multicolumn{4}{|c|}{ Number of hits per database } \\
\hline & PubMed-NCBI & $\begin{array}{l}\text { The Cochrane } \\
\text { library }\end{array}$ & Elsevier & Total \\
\hline Thyroid Gland & 74530 & 22 & 418 & 74970 \\
\hline Goiter & 37496 & 8 & 69 & 37573 \\
\hline Hypoxia-Inducible Factor & 19905 & 11 & 21 & 19937 \\
\hline HIF & 17272 & 6 & 17 & 17295 \\
\hline Middle east & 161253 & 22 & 314 & 161589 \\
\hline Iraq & 11819 & 1 & 83 & 11903 \\
\hline Thyroid Gland AND (“Hypoxia-Inducible Factor” OR HIF) & 18 & 0 & 0 & 18 \\
\hline $\begin{array}{l}\text { Thyroid Gland AND (“Hypoxia-Inducible Factor” OR HIF) AND "Middle } \\
\text { East” }\end{array}$ & 0 & 0 & 0 & 0 \\
\hline Thyroid Gland AND (“Hypoxia-Inducible Factor” OR HIF) AND Iraq & 0 & 0 & 0 & 0 \\
\hline $\begin{array}{l}\text { Thyroid Gland AND (“Hypoxia-Inducible Factor" OR HIF) AND } \\
\text { (Iraq OR "Middle East") }\end{array}$ & 0 & 0 & 0 & 0 \\
\hline Goiter AND (“Hypoxia-Inducible Factor” OR HIF) & 9 & 1 & 0 & 10 \\
\hline Goiter AND ("Hypoxia-Inducible Factor" OR HIF) AND "Middle East" & 0 & 0 & 0 & 0 \\
\hline Goiter AND (“Hypoxia-Inducible Factor” OR HIF) AND Iraq & 0 & 0 & 0 & 0 \\
\hline $\begin{array}{l}\text { Goiter AND (“Hypoxia-Inducible Factor" OR HIF) AND } \\
\text { (Iraq OR “Middle East") }\end{array}$ & 0 & 0 & 0 & 0 \\
\hline Total Number of Hits & 322302 & 71 & 922 & 323295 \\
\hline
\end{tabular}

of HIF-1 target genes depends on tissue type, lesion type and the co-expression of HIF-2. ${ }^{19-21}$ Studies concerning the role of HIFs in thyroid tissue are limited in the published body of literature. Additionally, there are even very few studies which looked at HIFs isoforms co-expressions in the same tissue specimens. Moreover, no single study to date investigates the expression of both isoforms in multinodular goitres and in connection with clinical toxicity. Therefore, we examined whether there is any difference in the co-expression of HIF- $1 \alpha$ and HIF- $2 \alpha$ between toxic and non-toxic multinodular thyroid goitres.

As stated earlier, most of the patients were females in their fifth decade of life, and the majority were clinically non-toxic. This finding goes in harmony with the usual epidemiological pattern of thyroid diseases, notably multinodular goitres that are more common in adult females as documented by Castro and Gharib in 2005, AlRrawak and co-workers in 2009, Mahdi and colleagues in 2010, and Mandel in 2014. ${ }^{22-25}$ Furthermore, our research confirmed that there is no significant difference between males and females in connection with IHC scoring of the investigated hypoxia-inducible factors.

Interestingly, our study demonstrated for the first time a significantly positive correlation between HIF-1 expression in multinodular thyroid goitre and the age as an independent demographic variable. To date, there were no studies that could explain the molecular mechanisms behind this positive and significant association. On the other hand, we found that age is not positively correlated with IHC scoring of HIF-2. However, there was a strong correlation in between IHC scoring of HIF-1 and IHC scoring of HIF-2. This finding is logically expected as the role of hypoxia-inducible factors are inherently intermingled at the molecular and cellular levels. The results also go in line with prior research attempts by Toschi and co-workers in 2008 and Liu and Xing in 2016. ${ }^{26,27}$ In 2010, Burrows and co-authors proposed that tissue genotype and the local microenvironment influence the bio-regulation of HIF proteins. ${ }^{28}$

Our study may have limitations including the lack of a control group and the relatively small sample size. Additionally, all cases were selected from a single hospital rather than being a true representative of the studied population by being based on a multi-centre study. Nevertheless, our research ranks well within the pyramid of the hierarchy of evidence-based Medicine as it is estimated to be of level-3 of the scheme established by the Oxford Centre for Evidence-Based Medicine as of May 2016. ${ }^{10}$

\section{CASE REPORTS OF INTEREST}

\section{Case-1}

A sixty years old male of the Arab ethnicity from Baghdad. $\mathrm{He}$ is an active person, athletic with a body-mass index (BMI) of $24.22 \mathrm{Kg} / \mathrm{m}^{2}$, and completely healthy apart from having benign prostate hyperplasia (BPH) which is controlled well via conservative medical treatment. A few months earlier, he developed a persistent sore throat and flu-like symptoms for one week for which he consulted an otolaryngologist. During the routine physical examination, the surgeon discovered an enlarged thyroid gland and nodule within the left lobe, and he advised the patient to perform thyroid function tests (TFT) and transcutaneous ultrasonography (US) of the neck to exclude any suspicious pathology affecting the thyroid gland, nearby lymph nodes, as well as a possibly infected branchial cyst. Thyroid function 
tests were within the standard acceptable limits with TSH level of $0.966 \mathrm{mIU} / \mathrm{L}$ while the US revealed an irregular solitary heterogenous thyroid nodule measuring 1.2 by $3.4 \mathrm{~mm}$ in diameter and occupying the inferior portion of the left thyroid lobe. The nodule was suspicious due to its mixed echogenicity and the existence of foci of intralesional calcifications. However, the remaining tissue of the thyroid gland, including the thyroid isthmus, was completely healthy.

Subsequently, radio-isotope scanning or fine needle aspiration cytology (FNAC) were advised for the patient to be correlated with clinical and biochemical findings to exclude any potential malignant transformation within the nodule. The cytology report of FNAC confirmed the presence of a moderate amount of colloid fluid with scattered follicular cells altogether with degenerative changes and foci of calcifications. These findings favour a hyperplastic thyroid nodule for which a regular follow-up is mandatory. The patient then re-consulted the otolaryngologist and a general surgeon. Both advised to do a surgical removal of thyroid within the next two months and to do a thorough histological analysis of the entire resected thyroid tissue including the hyperplastic thyroid nodule. The general surgeon further recommended to carry out a total thyroidectomy rather than a single lobe resection. He justified his decision by saying "Better to do the total removal, the nodule might turn to be malignant on post-operative result, microcalcifications in the module is worrisome, and there is a chance that the patient will develop new nodules in the remaining part if he did a lobe removal only. It is always better to do total thyroidectomy; this is the international consensus".

The patient agreed to do a total thyroidectomy, and the surgery was carried out with success. However, the postoperative histological analysis of thyroid tissue proven the absence of malignant transformation. Following the operation, the patient recovered well post-operatively, and there were no complications concerning injury (laryngeal nerves), hematoma formation, speech, or electrolyte homeostasis. He was discharged two days following the surgery. He started hormone replacement therapy with thyroxine to compensate for the absent thyroid gland. Biochemical analysis was entirely within the accepted range including the complete blood count, differential WBC count, clotting factors and coagulation studies (PT, PT'T, INR), serum electrolyte levels (corrected serum Calcium, Magnesium, and Phosphorous), thyroid function tests, anti-thyroglobulin antibodies (Anti-TG Abs), vitamin D level, and parathormone (PTH) level. Ten days following the operation, the surgical stitches were removed, and the wound was clean with a beautiful cosmetic surgical scar. The take-home message from our case scenario is the potential benefit of using an intraoperative frozen section consultation. Unfortunately, this option was not available for the patient within the facilities of the healthcare system where he received medical attention. Intraoperative frozen sections can exclude neoplastic transformations while the patient is still on the surgical operating table. In case the diagnosis of malignancy was eliminated, the surgeon could have conveniently performed a single lobe removal rather than a total thyroidectomy that mandates the patient to be on hormonal supplements day-to-day and for the rest of his life.

\section{Case-2}

A thirty-five years old female of the Arab ethnicity from Baghdad. She complained of a cervical swelling with a persistent fever, fatigue and general unwell-being for more than three weeks for which she made a medical consultation. Physical examination shows a nodular enlargement of the thyroid gland that was also tender and hard in consistency, the presence of some palpable cervical lymph nodes, in association with high-grade fever and tachycardia. Laboratory investigations revealed mild leucocytosis with markedly elevated ESR. Thyroid function tests were within the accepted range. Ultrasonography of the thyroid gland revealed marked enlargement of the thyroid though no areas of haemorrhage or calcification were observed. Her surgeon advised doing FNAC which showed pathological features suggesting the diagnosis of papillary thyroid carcinoma. Accordingly, the surgeon decided to do a total resection of the thyroid. Histopathological study of the resected thyroid tissue confirmed the pathology as a case of non-specific inflammatory thyroiditis while excluding the malignant transformation suggested earlier via FNAC. The patient is now on daily $0.1 \mathrm{mg}$ tablets of thyroxine. The critical element of interest here is the potential diagnostic error based on FNAC without a proper representative tissue biopsy. Aspiration cytology not only lack fully-proof sensitivity and specificity, but it should be conducted with precision by an experienced cytopathologist under adequate guidance by ultrasonography. Moreover, inflammatory thyroiditis frequently present with an active inflammation affecting the entire gland that can easily trick the unexperienced pathologist into the diagnosis of papillary thyroid cancer. The accuracy of FNAC, as mentioned in case-1, can be supremely enhanced when combined with intra-operative frozen section technologies.

\section{ACKNOWLEDGEMENTS}

None

\section{CONFLICTS OF INTEREST}

The authors have no competing interests to be declared. 


\section{SOURCE OF FUNDING}

This study is entirely self-funded.

\section{REFERENCES}

1. Zhao W, Han C, Shi X, Xiong C, Sun J, Shan Z, Teng W. Prevalence of goiter and thyroid nodules before and after implementation of the universal salt iodization program in mainland China from 1985 to 2014: a systematic review and meta-analysis. PloS one. 2014 Oct 14;9(10): e109549.

2. Gandolfi PP, Frisina A and Raffa M. The incidence of thyroid carcinoma in multinodular goiter: retrospective analysis. Acta Bio Medica Atenei Parmensis 2004; 75(2):114-117.

3. Brahimi-Horn MC, Chiche $\mathrm{J}$ and Pouysségur J. Hypoxia and cancer. Journal of Molecular Medicine 2007; 85(12):1301-1307.

4. Ke $\mathrm{Q}$ and Costa M. Hypoxia-inducible factor-1 (HIF-1). Molecular Pharmacology 2006;70(5):1469-1480.

5. Vooijs MA, Gort EH, Groot AJ, der Wall EV and van Diest PJ. Hypoxic regulation of metastasis via hypoxia-inducible factors. Current Molecular Medicine 2008;8(1):60-67.

6. Rankin EB and Giaccia AJ. The role of hypoxia-inducible factors in tumorigenesis. Cell death and differentiation 2008; 15(4):678.

7. Majmundar AJ, Wong WJ and Simon MC. Hypoxia-inducible factors and the response to hypoxic stress. Molecular cell 2010; 40(2):294-309.

8. Luna LG. Manual of histologic staining methods of the Armed Forces Institute of Pathology. Armed Forces Institute of Pathology (US). Armed Forces Institute of Pathology (US).; 1968.

9. Munro BH. Manual of Histologic Staining Methods of the Armed Forces Institute of Pathology. Pathology1971; 3(3):249.

10. Centre for Evidence-Based Medicine (CEBM, University of Oxford). OCEBM Levels of Evidence. https://www.cebm. net/2016/05/ocebm-levels-of-evidence/(accessed 17 July 2018).

11. Zeng $X$, Zhang $Y$, Kwong JS, Zhang $C$, Li S, Sun F, Niu Y and $\mathrm{Du} \mathrm{L}$. The methodological quality assessment tools for preclinical and clinical studies, systematic review and meta-analysis, and clinical practice guideline: a systematic review. Journal of Evidence-based Medicine 2015;8(1):2-10.

12. Greenhalgh T, Howick $\mathrm{J}$ and Maskrey N. Evidence based medicine: a movement in crisis?. BMJ 2014; 348:g3725.

13. Schito L, Semenza GL. Hypoxia-inducible factors: master regulators of cancer progression. Trends in cancer. 2016 Dec 31;2(12):758-70.

14. Jóźwiak P, Ciesielski P, Zaczek A, Lipińska A, Pomorski L, Wieczorek M, et al. Expression of hypoxia inducible factor $1 \alpha$ and $2 \alpha$ and its association with vitamin $C$ level in thyroid lesions. Journal of Biomedical Science 2017; 24(1):83.

15. Smith TG, Robbins PA and Ratcliffe PJ. The human side of hypoxia-inducible factor. British Journal of Haematology 2008; 141(3):325-334.

16. Eales KL, Hollinshead KE and Tennant DA. Hypoxia and metabolic adaptation of cancer cells. Oncogenesis 2016; 5(1):e190.

17. Loboda A, Jozkowicz A and Dulak J. HIF-1 versus HIF-2-is one more important than the other?. Vascular pharmacology 2012; 56(5-6):245-251.

18. Bersten DC, Sullivan AE, Peet DJ and Whitelaw ML. bHLH-PAS proteins in cancer. Nature Reviews Cancer 2013; 13(12):827.

19. Koh MY, Lemos R, Liu XP and Powis G. The Hypoxia-Associated Factor Switches Cells from HIF-1 $\alpha$ - to HIF-2 $\alpha$-Dependent Signaling Promoting Stem Cell Characteristics, Aggressive Tumor Growth and Invasion. Cancer Research 2011; 71(11): DOI: 10.1158/0008-5472.CAN-10-4142.

20. Abd-Aziz N, Stanbridge EJ and Shafee N. Bortezomib attenuates HIF-1-but not HIF-2-mediated transcriptional activation. Oncology letters 2015;10(4):2192-2196.

21. Påhlman S, Lund LR and Jögi A. Differential HIF-1 $\alpha$ and HIF-2 $\alpha$ expression in mammary epithelial cells during fat pad invasion, lactation, and involution. PLoS One 2015;10(5):e0125771.

22. Mandel SJ. A 64-year-old woman with a thyroid nodule. JAMA 2004; 292(21):2632-2642.

23. Mahdi QA, Ahmed BS and Kadhim MA. The frequency of thyroid carcinoma in patients with solitary and multiple nodules utilizingultrasound guided fine needle aspiration cytology (FNAC): A prospective study (Thyroid carcinoma and U/S guided FNA). Journal of the Faculty of Medicine2010; 52(2):136-140.

24. Castro MR and Gharib $\mathrm{H}$. Continuing controversies in the management of thyroid nodules. Annals of Internal Medicine 2005; 142(11):926-931.

25. Al-Rrawak K, Al-Sarraf SA and Sulaiman TI. Changing Patterns of Thyroid Pathology and Trends of Surgical Treatment. Journal of the Faculty of Medicine 2009; 51(1):12-16.

26. Liu R and Xing M. TERT promoter mutations in thyroid cancer. Endocrine-related cancer 2016:ERC-15.

27. Toschi A, Lee E, Gadir N, Ohh M and Foster DA. Differential dependence of hypoxia-inducible factors $1 \alpha$ and $2 \alpha$ on mTORC1 and mTORC2. Journal of Biological Chemistry 2008; 283(50):34495-34499.

28. Burrows N, Resch J, Cowen RL, von Wasielewski R, Hoang-Vu C, West CM, et al. Expression of hypoxia-inducible factor $1 \alpha$ in thyroid carcinomas. Endocrine-related Cancer 2010;17(1):61-72.

\section{Authors Contribution:}

MA-Study design, data collection and tabulation, histopathological analyses, and review of literature; SE- Study design, Supervision of the study, review of the literature, and editing the first draft of the manuscript; AAI- Data tabulation, statistical analyses, and writing the first draft of the article; BA- Conducted histopathological studies including immune histochemical procedures.

Work Attributed to: Department of Anatomy, Histology, and Embryology, College of Medicine, Al-Mustansiriya University, Iraq.

Orcid ID:

Dr. Ahmed Al-Imam - (D https://orcid.org/0000-0003-1846-9424

Dr. Mohammed Assi - ib https://orcid.org/0000-0002-9852-8799

Source of Support: Nil, Conflict of Interest: None declared. 